\title{
SPATIAL INTEGRATION ANALYSIS OF PROVINCIAL HISTORICAL AND CULTURAL HERITAGE RESOURCES BASED ON GEOGRAPHIC INFORMATION SYSTEM (GIS)—_A CASE STUDY OF SPATIAL INTEGRATION ANALYSIS OF HISTORICAL AND CULTURAL HERITAGE RESOURCES IN ZHEJIANG PROVINCE ${ }^{1}$
}

\author{
Wen Luo ${ }^{\text {a }}$, Jie Zhang ${ }^{\text {a }}$, Qilin Wu ${ }^{\text {a }}$, Jie Chen ${ }^{\text {a }}$, Xiaowei Huo ${ }^{\text {a }}$, Jie Zhang ${ }^{\text {a }}$, Yunsi Zhang ${ }^{\text {a }}$, Tong Wang ${ }^{\text {a }}$
}

a Tsinghua Tongheng Urban Planning and Design Institute, Research Center for Heritage Conservation and Urban-Rural Development,100085 Beijing, China-( luowen, zhangjie02, wuqilin, chenjie1, huoxiaowei, zhangjie3, zhangyunsi, wangtong)@ thupdi.com

KEY WORDS: GIS Spatial Integration Analysis Historical and Cultural Heritage Resources

\begin{abstract}
:
In China historical and cultural heritage resources include historically and culturally famous cities, towns, villages, blocks, immovable cultural relics and the scenic spots with cultural connotation. The spatial distribution laws of these resources are always directly connected to the regional physical geography, historical development and historical traffic geography and have high research values. Meanwhile, the exhibition and use of these resources are greatly influenced by traffic and tourism and other plans at the provincial level, and it is of great realistic significance to offer proposals on traffic and so on that are beneficial to the exhibition of heritage resources based on the research of province distribution laws.

This paper takes the spatial analysis of Geographic Information System (GIS) as the basic technological means and all historical and cultural resources in China's Zhejiang Province as research objects, and finds out in the space the accumulation areas and accumulation belts of Zhejiang Province's historic cities and cultural resources through overlay analysis and density analysis, etc. It then discusses the reasons of the formation of these accumulation areas and accumulation belts by combining with the analysis of physical geography and historical geography and so on, and in the end, linking the tourism planning and traffic planning at the provincial level, it provides suggestions on the exhibition and use of accumulation areas and accumulation belts of historic cities and cultural resources.
\end{abstract}

\section{INTRODUCTION}

In the previous researches on historically and culturally famous cities, towns and villages and so on, researchers often regard these heritages as a "sphere" to study its form, function, operation mode, and space layout. But if we look upon all these cultural resources as "points", especially when we mark all cultural resources within a certain range on the map as "points", we can see more information of space layout. The spatial distribution laws of these cultural resources at large scales are often directly linked to the regional physical geography, historical development and historical traffic geography and are of high research values.

On the other hand, in recent years, with the spread of concepts like cultural route and heritage corridor and so on, the protection and utilization of China's cultural heritages increasingly tend to regional collaboration and regional integration. For a province with wide space and multitudinous heritages, the exhibition and use of cultural resources are greatly influenced by province traffic and tourism and other plans, and it is of strong realistic significance to offer proposals on traffic and so on that are beneficial to the exhibition of heritage resources based on the research of province distribution laws.

Zhejiang Province is one of the districts with highly developed economy and culture in China since the Southern Song Dynasty
(1127-1279), and it is abundant in historical and cultural resources. This paper uses the technology of GIS to analyze the distribution status of historical and cultural resources in the whole Zhejiang Province, aiming to reveal the spatial distribution laws of the heritages resources in this region so as to make proposals to the province-level management and rational planning and utilization of heritages resources.

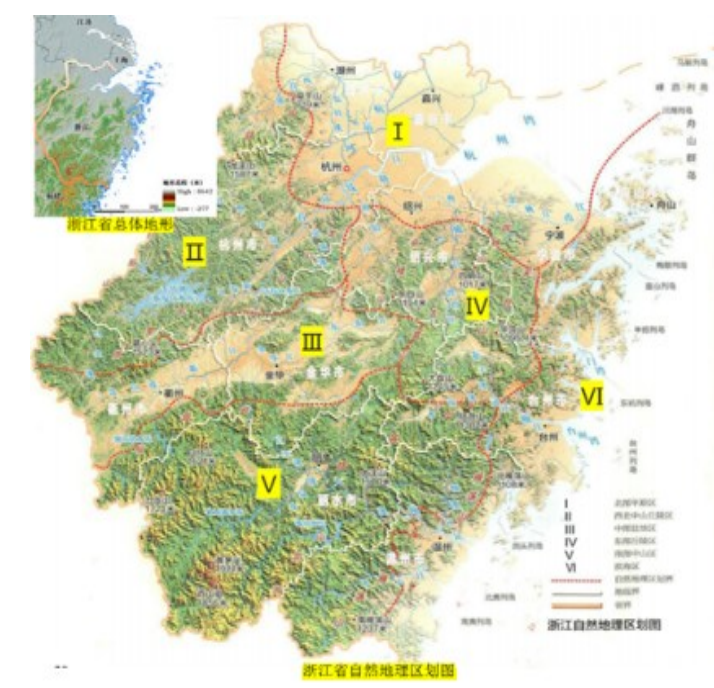

1 This paper is supported by the research of “A study on the potential estimation of Zhejiang Province's historically and culturally famous cities" trusted by the Housing and Urban-Rural Construction Office of Zhejiang Province 
I : Northern Plain Area

II : Northwestern Middle-Elevation Mountain and Hills Area

III: Central Basin Area

IV: Eastern Hills Area

V: Southern Middle-Elevation

Mountain and Hills Area

VI: Coastal Area

Figure 1. Topographic Maps of Zhejiang Province (Accessed at: Internet)

\section{ZHEJIANG HISTORICAL AND CULTURAL RESOURCE DISTRIBUTION AND DENSITY ANALYSIS}

\subsection{General situation of Zhejiang}

Zhejiang is located in the south wing of the Yangtze River Delta of the southeast coastal region in China. With a land area of 105.5 thousand square kilometers, it is one of China's smaller provinces Zhejiang has a complex terrain, tilting from southwest to northeast, and it can be divided into 6 regions including the plain in northern Zhejiang, middle-elevation mountain and hills area in western Zhejiang, hills in eastern Zhejiang, Jin-qu basin in the middle of Zhejiang Province, mountainous region in the southern Zhejiang and southeast coastal plain and coastal islands. The eight water systems consisting of Qiantang River, Ou River and Ling River, etc., and the Zhejiang section of Grand Canal flows through the province, forming natural ducts for regional communication. With four distinctive seasons and plentiful rainfall, Zhejiang belongs to subtropical monsoon climate and is one of the districts with highly developed economy and culture in China since the Southern Song Dynasty. It is the typical Jiangnan Watertown and is known as the "Home of Silk" and "land of fish and rice".

Zhejiang possesses a sea of historical and cultural resources, and its protection and management level of cultural heritages is also at the top of the list in China. Currently Zhejiang in total has 9 national historical and cultural cities, ranking third, next only to Jiangsu and Shandong. In the third national archaeological survey, with over 73 thousand cultural relics, Zhejiang has the richest "family property" and has 8 thousand more relics than Henan Province (the second place) does.

\subsection{Density analysis of Zhejiang historical and cultural resources}

In China, historical and cultural heritage resources include historically and culturally famous cities, towns, villages, blocks, immovable cultural relics and the scenic spots with cultural connotation. The research scale is the whole Zhejiang Province, so we screened the levels of its historical and cultural resources, mainly investigating the national-level and provincial-level historical and cultural cities, towns, villages, scenic spots and blocks with relatively higher values and greater influence and the Chinese traditional villages and major historical and cultural sites protected at the national level.

We firstly imported all resource points in Zhejiang Province into GIS according to longitudes and latitudes, made weighting and assignment on the basis of the levels and values of resources, and then obtained the Zhejiang provincial cultural resource distribution and density map.

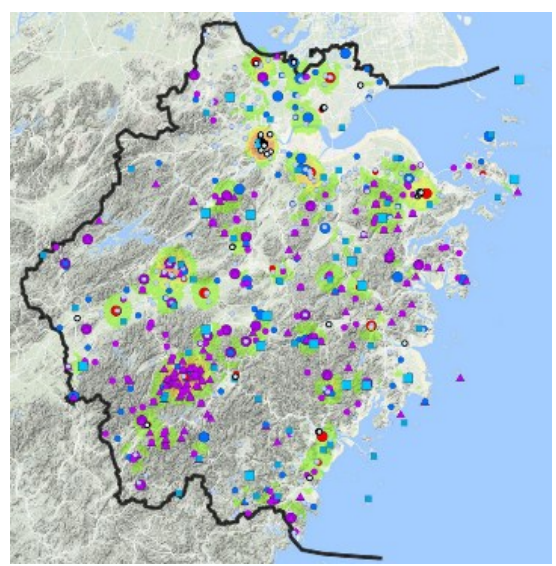

- state level historical and cultural city

- state level historical and cultural town

- state level historical and cultural village

state level scenic spots

Chinese traditional village

- provincial level historical and cultural city

- provincial level historical and cultural town

- provincial level historical and cultural village

provincial level scenic spots state level historical and cultural block

provincial level historical and cultural block key national heritage conservation unit - Zhejiang province boundary

Low degree of gathering High degree of gathering

Figure 2.Distribution and Density Map of Cultural Resources

\subsection{Correlation analysis of Zhejiang historical and cultural resource density}

If the density analysis chart is superimposed on the administrative city map of Zhejiang Province, it shows that the distribution density of Zhejiang historical and cultural resources is related to the distribution of administrative cities. In Zhejiang northern region, especially, administrative central cities in general are the cores in historical and cultural resource distribution. It is because Zhejiang modern administrative central cities develop basically from historical central cities, and these cities have profound economic and cultural accumulation and abundant cultural resources.

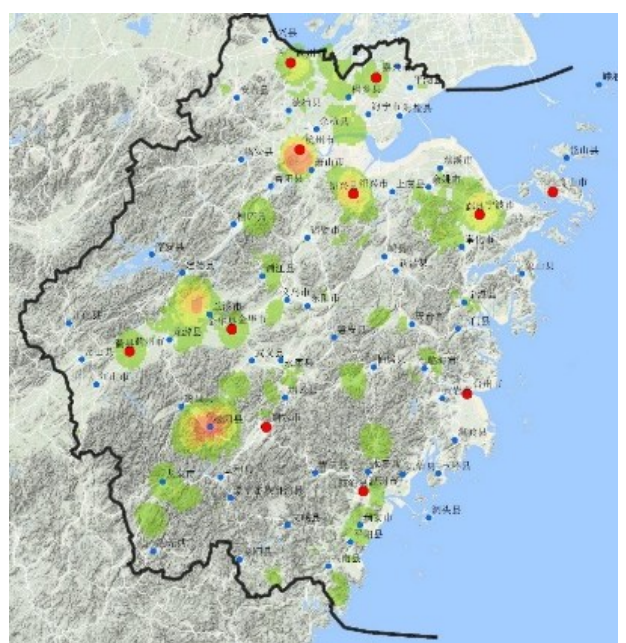




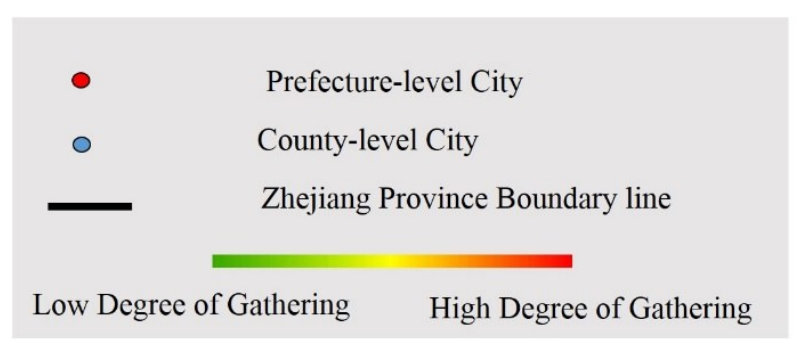

Figure 3. The Gathering Map of the Culture Resources Density and Districts in Zhejiang Province

If the density analysis chart is superimposed on the topographic map and drainage map of Zhejiang Province, it can be seen that the areas in Zhejiang with dense cultural resources are in the riverside and especially in river confluence reaches. Therefore, the river, as the natural and traffic resource that is necessary for human existence and development, plays a fundamental role and tandem role.

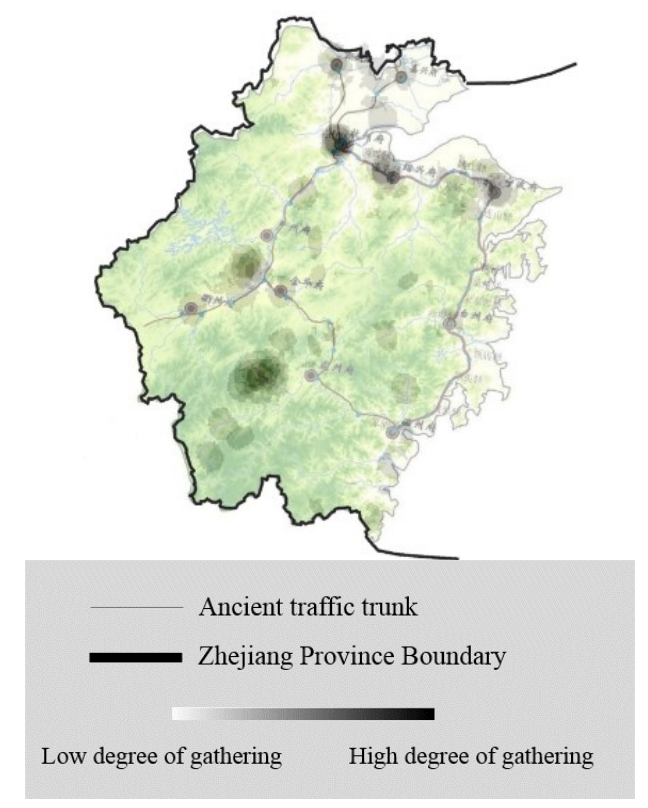

Figure 4.Overlay map of cultural resources and ancient traffic

It is worth noting that in the density analysis chart Zhejiang southwestern mountainous area cored with today's Songyang County has a large-scale dense district which strays from major central cities and the historical traffic line. After examining the data we can find that, on the one hand, it is because Songyang County does reserve a large quantity of cultural resources and especially Chinese traditional villages, and on the other hand, this profits from the Songyang County government's high attention to this work. Songyang in 2015 was awarded as China's first demonstration county of traditional village protection and development, which further motivated Songyang's protection work of traditional villages. Hence the density is impacted by certain policies and human factors, and its inherent laws are distinct from other patch densities.

\section{ACCUMULATION AREAS AND ACCUMULATION BELTS OF ZHEJIANG HISTORICAL AND CULTURAL RESOURCES}

Through superimposition analysis it can be observed that the patches of higher historical and cultural resource density in
Zhejiang have their own internal logic and reasons, and according to the density and internal logic we get 6 accumulation areas and 4 accumulation belts of Zhejiang historical and cultural resources, which are:

Accumulation areas:

a. Songyang cultural resources concentrated area, themed with the traditional villages characterized by the southern Zhejiang. b. Hangzhou cultural resources concentrated area, themed with the ancient capital and human landscapes of West Lake.

c. Lanxi-Jiande cultural resources concentrated area, themed with the construction landscape characterized by the western Zhejiang. d. Shaoxing cultural resources concentrated area, themed with the Yue culture and modern celebrity culture.

e. Ningbo cultural resources concentrated area, themed with the Hemudu Site and sea silk port culture.

f. Huzhou cultural resources concentrated area, themed with the canal culture and waterside culture.

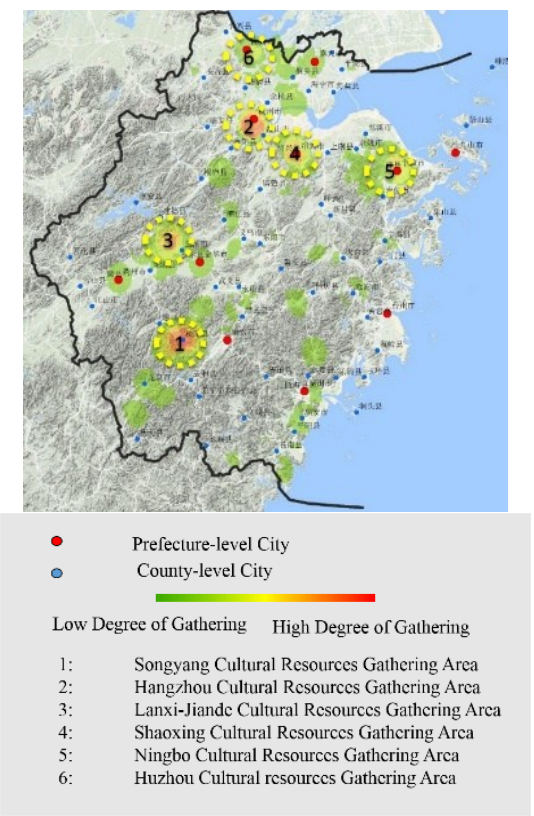

Figure 5. Gathering Map of Culture Resources Density and Districts in Six Areas

Accumulation belts:

a. Hangjiahu cultural resource accumulation belt. It is connected by the network of rivers and has three subject areas which are Hangzhou, Jiaxing and Huzhou. Among them, Huzhou area is themed with the Jiangnan (regions south of the Yangtze River) town culture, Jiaxing area is themed with the canal culture, and Hangzhou area is themed with the capital culture.

b. Eastern Zhejiang canal cultural resource accumulation belt. It is joined by the canal in the eastern region of Zhejiang Province, and three are two subject areas of Ningbo and Shaoxing within it. Ningbo area is themed with the sea silk port culture, while Shaoxing area is themed with the Yue culture and modern celebrity culture.

c. Jingqu Corridor cultural resource accumulation belt. It is connected by the transport lines of Jingqu Corridor and has three subject areas which are Jinghua, Quzhou and LanxiJiande. Jinghua area is themed with the traditional business culture, Quzhou area is themed with the Confucius southern 
culture, and Lanxi-Jiande area is themed with the construction landscape characterized by the western Zhejiang.

d. Upper Ou River cultural resource accumulation belt. It is linked by the upper streams of Ou River. It has three subject areas, and they are Songyang, Longquan and YongkangWuyi-Jinyun, among which Songyang area is themed with the traditional villages characterized by the southern Zhejiang, Longquan area is themed with the celadon culture, and Yongkang-Wuyi-Jinyun area is themed with the construction landscape characterized by the central Zhejiang.

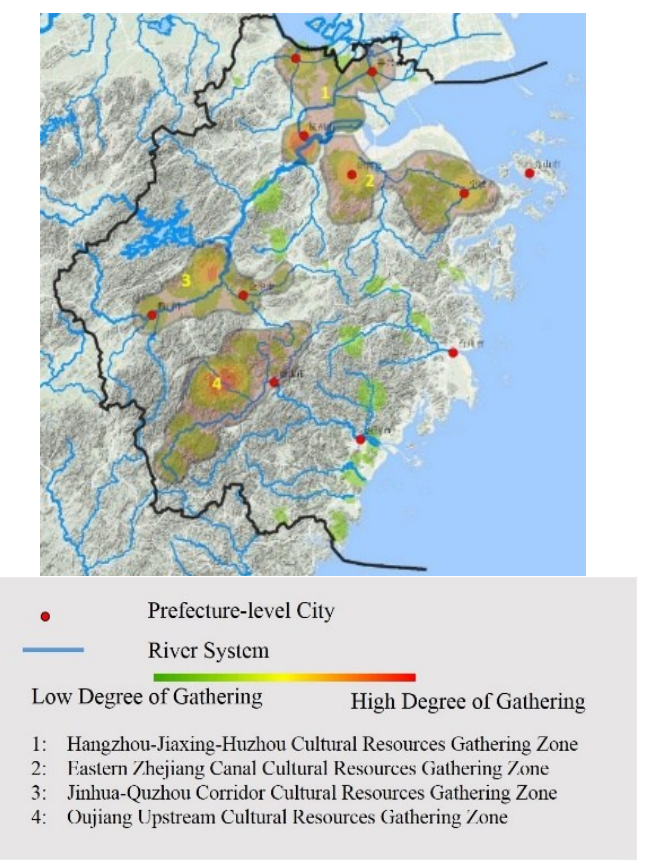

Figure 6. Gathering Map of Culture Resources Density and Districts in Four Zones

\section{SUGGESTIONS ON THE EXHIBITION AND USE OF CULTURAL RESOURCE ACCUMULATION AREAS AND ACCUMULATION BELTS}

\subsection{Suggestions on the exhibition and use of provincial lineal or serial cultural heritages}

The vital lineal or serial cultural heritages within Zhejiang Province include the Great Canal (section), Zhedong Canal, Tang Poetry Road, Zhejiang Coast Defense System, Seawall System, and Modern Railway and Porcelain Road and so on. Based on the above accumulation areas and accumulation belts and their resource themes, the cultural lines of Great Canal (section) and Zhedong Canal have basically been covered by the current accumulation areas and accumulation belts, and part of Modern Railway, Zhejiang Coast Defense, Seawall and Porcelain Road have mostly been covered by above accumulation areas and accumulation belts. But given the present (higher level) resource conditions, the themes have not been highlighted, so we propose to enhance resource excavation and systematic research. As the Tang Poetry Road has neither formed themes and systems in cultural sources, nor been noted by provincial town planning, we suggest that in addition to the strengthening of the research and excavation of internal cultural resources, it should rely on the numerous historic cities along Tang Poetry Road and take them as transit centers and service centers, and integrate with natural resources and Tianlao Mountain and Cao'e River and other tourist resources to comprehensively foster and build a lineal or serial cultural heritage site.

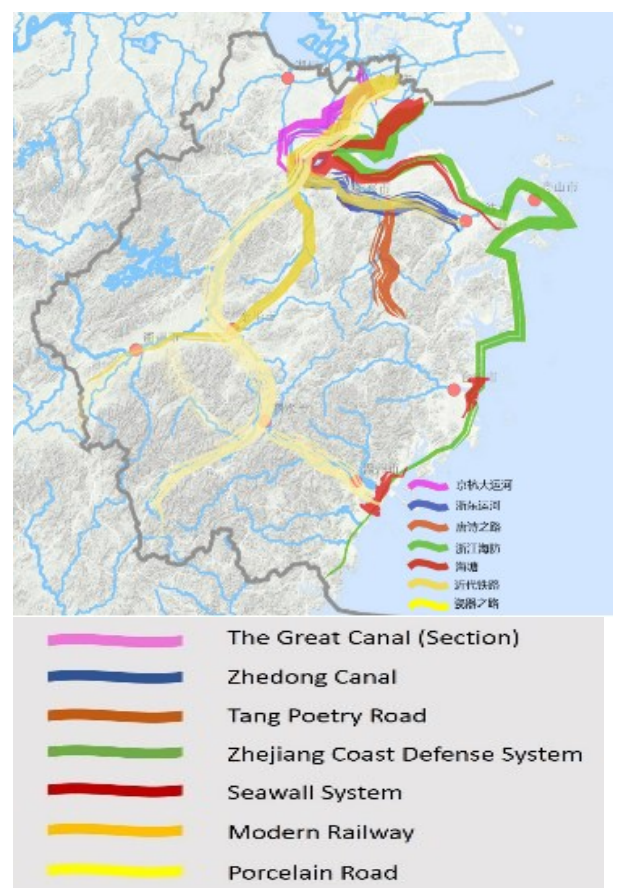

Figure 7. The Distribution of Lineal Cultural Heritages in Zhejiang Province

\subsection{Correctional proposals for provincial tourism planning}

The urban system planning of Zhejiang Province specifies its tourism development pattern of 'three belts and ten areas'. Combing with the analysis of above historical and cultural resource density and accumulation areas and belts, we suggest that the Zhejiang Province tourism pattern may make the following adjustments:

We suggest adding Tang Poetry Road cultural tourism belt and upper Ou River cultural tourism belt on the basis of the existing "three belts" which are Hangzhou Bay cultural leisure tourism belt, eastern Zhejiang coastal and marine tourism belt and southwestern Zhejiang landscape and ecological tourism belt.

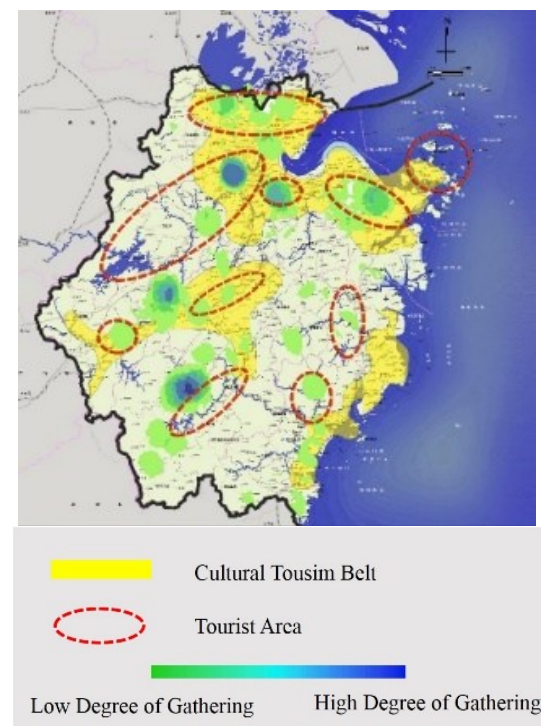


Figure 8. Culture Resources Density and Tourism Development Plan in Zhejiang Province

For 'ten areas', we recommend bringing the human landscape of Fuchun River into Hangzhou international leisure tourist area, adding the themes of Maritime Silk Road and modern cultures to Ningbo Hemudu-Dongqian Lake tourist area, increasing the scope of Wenzhou Yandang Mountains-Nanxi River tourist area and bringing Wenzhou, Ruian and Pingyang and other areas into it, absorbing Lanxi area into Jinhua business cultural tourist area, building Taizhou Tiantai Mountain and Shenxianju tourist areas by combining with the Tang Poetry Road, and bringing Songyang area and Longquan area into the Lishuilvgu custom tourist area.

\subsection{Proposals for provincial transport planning}

If the density distribution chart of historical and cultural resources is superimposed on the provincial traffic map, it shows that:

The spatial distribution of provincial air harbors primarily meets cultural tourism demands, but Jiaxing Airport and Lishi Airport have not yet opened and therefore need to promote the construction.

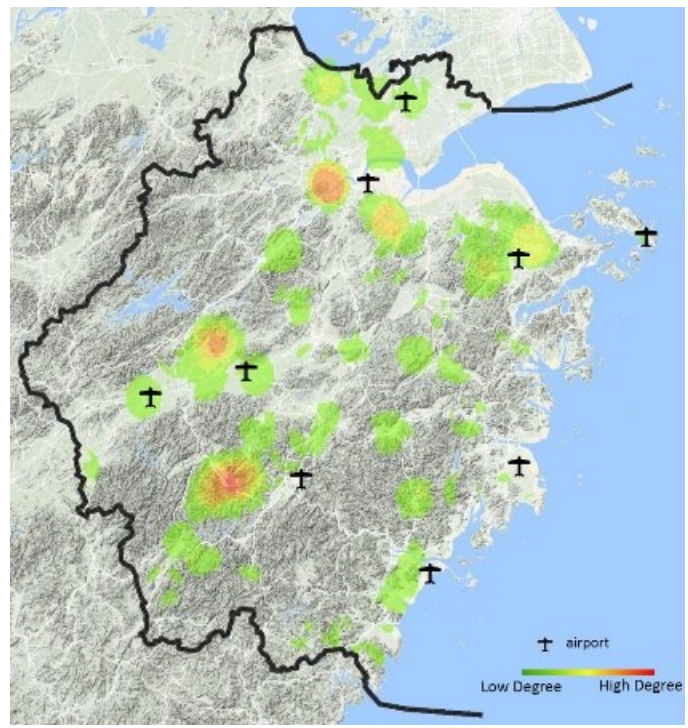

Figure 9. Overlay Map of Cultural Resources Density and Airports

Highways within Zhejiang Province basically connect the resource accumulation areas together, but the accumulation area between Jiande and Lanxijian has not yet been covered by highways.

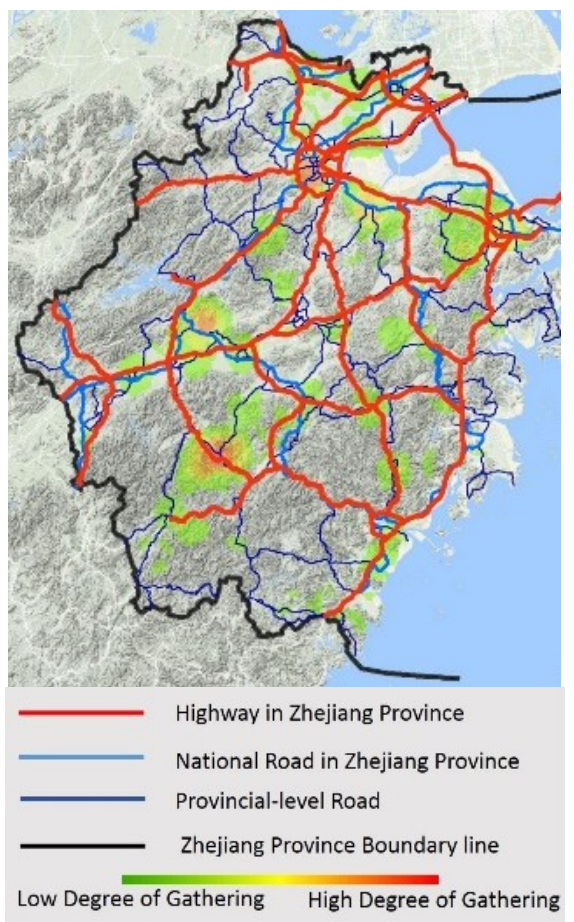

Figure 10 .The Gathering Map of Cultural Resource Density and Traffic Distribution

Railway lines connect the major central cities in series, but the Songyang accumulation area and Jiande-Lanxi accumulation area which have the densest cultural resources have not been covered.

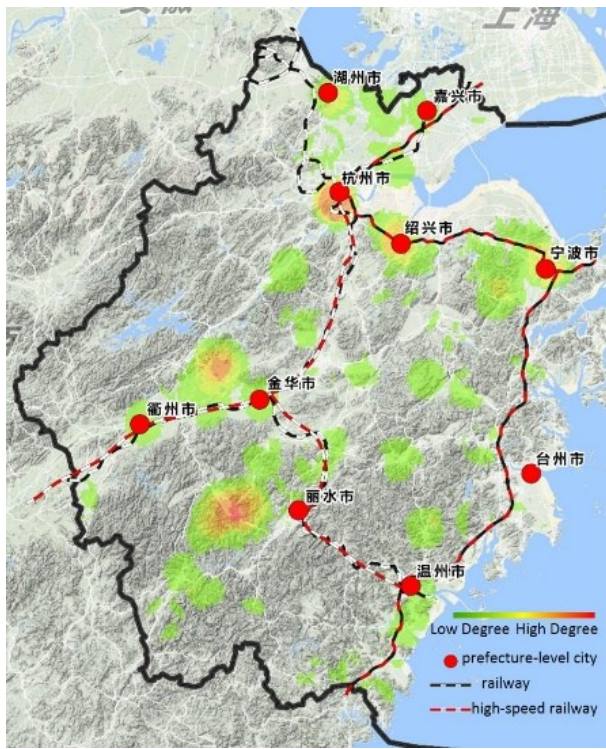

Figure 11. Overlay Map of Cultural Resources Density and Railways

In The 2011-2020 Urban System Planning of Zhejiang Province, all the above transportation problems have been added to the future planning. We suggest gradually improving transportation infrastructure construction in accordance with this Planning, and paying close attention or giving priority to the construction of Jiaxing Airport and Lishi Airport and also the construction of Quli Railway, Jinjian Railway and Hangjianqu Railway.

\subsection{Proposals for provincial transport planning}


If the density distribution chart of historical and cultural resources is superimposed on the provincial traffic map, it shows that:

The spatial distribution of provincial air harbors primarily meets cultural tourism demands, but Jiaxing Airport and Lishi Airport have not yet opened and therefore need to promote the construction.

Highways within Zhejiang Province basically connect the resource accumulation areas together, but the accumulation area between Jiande and Lanxijian has not yet been covered by highways.

Railway lines connect the major central cities in series, but the Songyang accumulation area and Jiande-Lanxi accumulation area which have the densest cultural resources have not been covered.

According to the 2011-2020 Urban System Planning of Zhejiang Province, all the above transportation problems have been added to the future planning. We suggest gradually improving transportation infrastructure construction in accordance with this Planning, and paying close attention or giving priority to the construction of Jiaxing Airport and Lishi Airport and also the construction of Quli Railway, Jinjian Railway and Hangjianqu Railway.

\section{REFERENCE:}

Guo,L., Li,J., and Huang,J.,2015, Study of Mobile GIS Application in the Census of Guangzhou City Cultural Heritage.Geomatics and Spatial Information Technology,38(8).

Mao, F., Zhou, W., Liang, Z., and Hu, Y. 2008, Research on the Application of Spatial Information Technology in the Conservation of Historical and Cultural Heritage. Journal of Urban and Regional Planning, 2008, 1(3).

PGOZP, (2011).The 2011-2020 Urban System Planning of Zhejiang Province.

Shenglin, L., 2014, Application of GIS for Cultural Heritage Conservation and Archaeological Research. Jianghan Archaeology, 3, pp.98-108.

Tu, C., 2006, Research on Application of GIS in Historical Cultural Resource Protection. Computer technology and development, 16(7). 